\title{
Learning about Learners: System Learning in Virtual Learning Environment
}

\author{
Zhengxin Chen
}

\begin{abstract}
Editorial Note: The program committee of ICVL 2007 sent to IJCCC nine of the best papers presented at the conference, with the recommendation to be published in an extended form. After a new evaluation, our reviewers decided that five of those papers can be published in IJCCC, two of which in this issue.
\end{abstract}

\begin{abstract}
Virtual learning is not just about a set of useful IT tools for learning. From an examination on where virtual learning stands in the overall learning spectrum, we point out the important impact of natural computing on virtual learning. We survey and analyze selected literature on important role of natural computing aspects, such as emergence (using swarm intelligence to achieve collective intelligence) and emotion, to virtual learning. In addition, in order to effectively incorporate these aspects into virtual learning, we propose using infrastructural support for virtual learning through system learning: The virtual learning environment not only provides facilities for learners, but also observes the behavior of learners and takes actions, so that its own performance can be improved (i.e., to better serve the learners). In this sense, system learning is concerned with learning about learners. Consequently, a virtual learning environment is a true human-machine symbiosis, paired by human learning and system learning.
\end{abstract}

Keywords: Natural computing, collective intelligence, emergence, emotion, system learning, virtual learning architecture

\section{Introduction}

As a software system, a virtual learning environment is intended to offer a virtual environment for learning where the learning process is based on information technology (IT). Virtual learning environment facilitates computerized learning or computer-enhanced learning (e-learning). Many projects in virtual learning have been designed to facilitate teachers in the management of educational courses for their students, especially by helping teachers and learners with course administration. The system can often track the learners' progress, which can be monitored by both teachers and learners. With advanced learning technology (ALT) it supports, virtual learning makes learning as a life-long journey easier to achieve than anytime else in history, and the entire world now becomes an open university.

However, although virtual learning has started producing fruitful results, fundamental issues related to virtual learning is seldom examined. Related to this is the pulic misconception that virtual learning is just a set of IT (information technology) tools or techniques. To address this problem, in this paper we first take a look at the learning spectrum, so that we can have a better understanding about where virtual learning stands in this big picture (Section 2). Since this examination reveals the importance of natural computing for virtual learning, we provide a brief review on natural learning (Section 3). We then examine two important features related to natural computing, namely, emergence and emotion, and review existing work relevant to virtual learning (Section 4 and Section 5). The introduction of natural computing in virtual learning necessities the concept of system learning; its important role is examined (Section 6). We conclude this paper by indicating the need for investigating a virtual learning architecture (or a framework) where human virtual learning is paired with system learning (Section 7). 


\section{The learning spectrum}

Virtual learning is concerned with IT assisted human learning and much of research work in virtual learning addresses various technical issues to enhance human learning. However, any discussion concerned with learning using contemporary IT techniques is incomplete if we do not take a look on the issue of machine learning (http://robotics.stanford.edu/people/nilsson/mlbook.html), a subfield in AI devoted to developing algorithms for enhanced performance of computers. Although virtual learning (for humans) and machine learning are two separate research areas, they are both related to learning with computers. So if we examine them together, we should have a better understanding about where virtual learning stands in the big picture.

This broad perspective raises an important issue: Virtual learning should not be simply viewed as just a set of IT tools (or techniques) to assist learning or education. It reminds us the importance of examining fundamental issues related to learning, such as nature of intelligence, various forms of intelligence, consciousness and thinking, as well as recent research progress related to brain and mind, and even study activities observed in various forms of life - not just humans, but animals as well, so long as they demonstrate a kind of learning ability. These studies could shed meaningful insight for developing better virtual learning environments. As noted in [16], in recent years educators have explored links between classroom teaching and emerging theories about how people learn. Recommended educational approaches, consist primarily of trying to maintain a relaxed, focused atmosphere that offers options for learning in individually satisfying ways. One thing we must be kept in mind is that the brain is complex and while research has revealed some significant findings, there is no widespread agreement about their applicability to the general population or to education in particular. Nevertheless, brain research provides rich possibilities for education [16]. Articles in the magazine Scientific American Minds (http://www.sciammind.com/) also shed interesting lights on brain/mind research and education.

The issue of studying various forms of learning activities leads to the next question: Is anything setting in between virtual learning and machine learning? Although this may be an open question which is subject to debate, we would like to offer a possible answer: learning from nature through natural computing, which is the computational version of the process of extracting ideas from nature to develop "artificial" (computational) systems ("artificial" means human-made). Since natural computing is aimed to model the nature or even compute with the nature, it looks for intellectual inspiration from all forms of life (not restricted to human beings) - such as flocks or ants. Since natural computing sets between the research of "full human" (i.e., virtual learning) and non-human (i.e., machine learning), it fits in the missing link in the learning spectrum. Amending the hierarchical diagram provided by [1], we have the learning hierarchy as shown in Figure 1. (Note: For more details on various forms within distance learning, refer to the original figure in [1]).

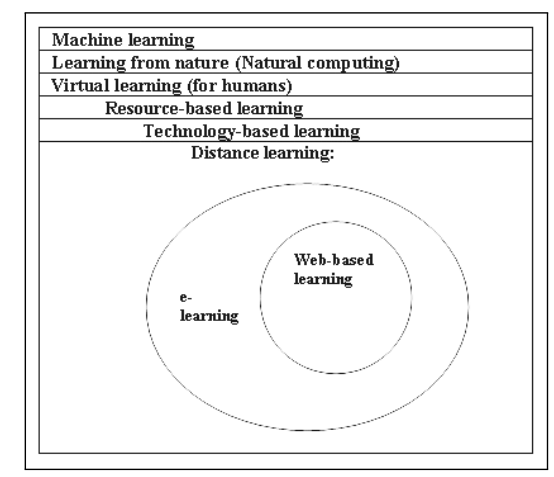

Figure 1: Learning spectrum from machine learning to various forms of virtual learning

But why should we bother natural computing at all in the context of virtual learning? The answer is 
simple: It may provide new interesting ideas not addressed in traditional learning or education context, so that virtual learning can benefit from that. Note that natural computing is not new to virtual learning community: As we are going to see soon, projects using various natural computing techniques have been conducted for virtual learning. Yet there is a need to conduct more systematic research on the rich impact of natural computing in virtual learning. Such kind of investigation will eventually benefit the study of natural computing as well, because the diverse applications in virtual learning extend the horizon of natural computing.

\section{Basics of natural computing}

In order to discuss how natural computing can help, first we have to provide a brief introduction on what natural computing is. There are numerous resources available for natural computing. For beginners, [4] provides a comprehensive coverage on the major fields with natural computing. The brief review in this section is based on that book.

The philosophy of natural computing lies in that most of computational approaches natural computing deals with are based on highly simplified versions of the mechanisms and processes present in the corresponding natural phenomena. Research work in natural computing can be grouped into three major categories, namely, computing inspired by nature, simulation and emulation of natural phenomena in computers, and computing with natural materials. Since the last one does not have direct impact on virtual learning (at least for now), we will not address it here.

The first category, computing inspired by nature, refers to making use of nature as inspiration for the development of problem solving techniques. The main idea is to develop computational tools (algorithms) by taking inspiration from nature for the solution of complex problems. The diverse areas (or approaches) under this category include evolutionary computing, neurocomputing, swarm intelligence, etc. Swarm intelligence refers to a property of systems of unintelligent agents of limited individual capabilities exhibiting collectively intelligent behavior, and has drawn attention from researchers to find useful applications in virtual learning (as to be briefly described later).

The second category, simulation and emulation of natural phenomena in computers, refers to a synthetic process aimed at creating patterns, forms, behaviors, and organisms that (do not necessarily) resemble "life-as-we-know-it." Its products can be used to mimic various natural phenomena, thus increasing our understanding of nature and insights about computer models. An interesting area under this line of research is artificial life, which is the study of man-made systems that exhibit behaviors characteristic of natural living systems. It has been hoped that by extending the empirical foundation upon which biology is based beyond the carbon-chain life that has evolved on Earth, artificial life can contribute to theoretical biology by locating life-as-we-know-it within the larger picture of life-as-it-could be.

Regardless of computing inspired by nature or simulation and emulation of natural phenomena in computers, there are several general concepts underlying various approaches in natural computing, such as agent, parallelism and distributivity, interactivity, adaptation, feedback, self-organization, emergence, etc. In addition, emotion is demonstrated not just in human beings, but many species of animals as well. Emotion has also been studied in artificial life.

Various natural computing concepts have already been used by authors working in virtual learning. Much effort has been put on researchers' "traditional" favorite such as adaptation. Yet successful employment of natural computing in virtual learning goes far beyond adaptation. Below we examine selected literature involving two crucial elements of natural computing and provide comments on their relevance to virtual learning: Emergence and emotion. 


\section{Exploring emergence for virtual learning}

Using simple terms, emergence can be defined as "a coming into view." However, emergence is definitely not a simple concept, and the computational study on emergence [6] is still in its infancy. As a typical demonstration of computational approach for emergence, swarm intelligence is a property of systems of unintelligent agents of limited individual capabilities exhibiting collectively intelligent behavior. Swarm intelligence includes any attempt to design algorithms or distributed problem-solving devices inspired by the collective behavior of social insects and other animal societies. Swarm intelligence is an emergent property of the swarm system as a result of principles of the five principles: proximity, quality, diversity, stability and adaptability. Two main lines of research in swarm intelligence are either based on social insects, or based on the ability of human societies to process knowledge [4].

The ability of ants to find short routes between nests and food sources suggests an approach to cost-effective, flexible and implementable wayfinding support. Paths identified by ants are not preplanned, but emerge, spontaneously, as a result of indirect communication between members of an ant colony - a form of indirect social navigation. Ants deposit a chemical substance called pheromone which can be sensed by other ants, thus achieving a kind of stigmergy, which refers to the process of indirect communication. This property can be very useful for virtual learning. In a virtual learning environment considered by [17], learners' interactions with learning resources and activities are recorded automatically as they progress through a body of knowledge. The time stamping of these interactions allows learning sequences to be identified which can be processed and aggregated to derive a given "pheromone strength" favoring paths along which more learners have been successful. This information can be fed back to other learners, providing a new source of navigational guidance indicating "good" ways through the body of knowledge - a self-organizing, stigmergic approach to wayfinding support.

In another experiment by [15], ant colony optimization (ACO) heuristics was applied to an e-learning problem: the pedagogic material of an online teaching Web site for high school students is modeled as a navigation graph where nodes are exercises or lessons and arcs are hypertext links. The arcs' valuation, representing the pedagogic structure and conditioning the Web site's presentation, is gradually modified through the release and evaporation of virtual pheromones that reflect the successes and failures of students roaming around the graph. A compromise is expected to emerge between the pedagogic structure as originally dictated by professors, the collective experience of the whole pool of students and the particularities of each individual. Collective behavior as demonstrated from ant colonies and simulated in computer programs exemplifies collective intelligence (or symbiotic intelligence), an intelligence that emerges from the collaboration and competition of many individuals - an intelligence that seemingly has a mind of its own. Yet emergence is not restricted in collective intelligence. In general, emergence refers to the way complex systems and patterns arise from a multiplicity of relatively simple interactions [4].

Two influential monographs on emergence should find profound impact on virtual learning: through a series of narratives to show complex adaptive systems that display emergent behavior governed by small sets of local rules, the discussion in [8] may shed useful intuitive thoughts on infrastructural support to achieve emergence in virtual learning, while [6] provides more technical insights on modeling issues of emergence in a more general, abstract setting, where emergence is explained through a reductionist perspective. We summarize system learning with the following features related to emergence. Exploring emergent properties using swarm intelligence and other techniques should be continued and strengthened for research virtual learning. Yet, current research related to emergence in virtual learning is largely confined in specific tasks. More systematic studies of the role of emergence in virtual learning are needed, particularly those related to the overall infrastructure of virtual learning. In addition, in the context of learning/education (including virtual learning), there is a need to distinguish emergence as a process (such as emerging ideas) from emergence as a product (such as an emerging pattern) - a feature which has not attracted enough attention it deserves. Here is a partial list of issues to be studied about emergence related to virtual learning: 
Nature of emergence (relevant to learning), such as: what are the relationships and differences between emergence and other features related to learning and discovery?

What can be achieved through emergence? - In the case of emerging ideas:

o Specific creative task (such as construction of analogs through emergence for analogical problem solving)

What can be achieved through emergence? - In the case of emerging "products":

o Global solution (such as an optimization of student pedagogical path);

o Solution for individuals (learning by taking advantage of emergence), such as how to come up with creativity thoughts in general.

In addition, there is a more general question: Is there a need to have a dedicated software component at system level to support emergence in a virtual learning environment? If yes, how to achieve this?

\section{Exploring emotion in virtual learning}

Recently the importance of emotion in education has drawn attention from researchers. For example, according to [16], educators may find the most useful information in research that focuses less on the physical and biochemical structure of the brain and more on the mind - a complex mix of thoughts, perceptions, feelings, and reasoning. Studies that explore the effects of attitudes and emotions on learning indicate that stress and constant fear, at any age, can circumvent the brain's normal circuits. A person's physical and emotional well-being is closely linked to the ability to think and to learn effectively. Emotionally stressful home or school environments are counterproductive to students' attempts to learn. While schools cannot control all the influences that impinge on a young person's sense of safety and well-being, classrooms and schools that build an atmosphere of trust and intellectual safety will enhance learning. Letting students talk about their feelings can help them build skills in listening to their classmates' comments. Finding ways to vent emotions productively can help students deal with inevitable instances of anger, fear, hurt, and tension in daily life. In an experimental study, the author of [12] interviewed eleven students studying online. These students identified emotions which were critical to their online learning.

In order to better understand where emotion stands in learning and education, it would be beneficial to take a look at the two books on general aspects of emotions with quite different emphases. From a psychological perspective, the experimental research of [9] showed that emotion can occur without cognitive processing in the cortex. In particular, we can learn some general principles of emotions by studying fear. In evolutionary terms, "fearless" animals would have been less likely to survive. The author further demonstrates that fear can be related to learning and fear learning is implicit. Although by no means we should endorse any kind of "learning through fear," this example does indicate emotion can have impact on education in a controlled manner, and with more secretes of emotion to be revealed in the future, some of the previously unknown principles involving emotions can be incorporated into virtual learning environment. Published one decade later, from a computational perspective, [10] aimed to establish a theory of how emotions get created. According to this theory, each emotional state is a different style of thinking. So there is no general theory of emotions, because the main idea is that each of the major emotions is quite different. For an adult person, the management is able to use these different ways of thinking very quickly as part of ordinary, common-sense thinking. What is the indication of this discussion to learning/education? The notion of emotion as a theory of thinking implies a potential opportunity to incorporate emotion into "mainstream" education theories, including those related to virtual learning. 
In a recent comprehensive volume directly address the issue of emotion in education [14], various theoretical perspectives on emotions in education have been examined, include the discussions on control-value theory of achievement emotions, self-regulation and social-constructivist learning, emotions as a main component of attributional theory, implications of goal-theory for achievement-related emotions, macro-cultural psychology, etc. The theoretical work is complemented with sets of studies on students' emotions in educational context, as well as teachers' emotions in educational contexts. The comprehensive of research on emotion in education as described in this volume sheds light for future work of dealing with the emotion factor in a virtual learning environment (even the book does not address issues directly related to virtual learning).

As the literature surveyed above shows, so far the important issue of emotion for education is still largely discussed at the traditional classroom setting. Nevertheless researchers have started addressing this issue in the context related to virtual learning. For example, [3] presents an analysis of the issues pertaining to computational emergence and emotion in (cognitive) agent systems and describes how a developing computational theory of cognition can be used to monitor and manage interactions with and within complex systems; this would harness unwanted and emergent states and behaviors before the computational system becomes dysfunctional. In another work, [13] describes a modular hybrid neural network architecture, called SHAME, for emotion learning. In addition, computational experiments on emotion also exist. For example, [7] proposes the architecture of learning companion agent with facial expression of emotion. Based on architectures referred to as $\mathrm{ABC}$ and ToK, the emotion agent architecture contains five modules to realize the interaction in the world. A particular part of this research is the transition between emotion space in emotion module and facial expression space in facial expression module. Although this work is not directly related to virtual learning, it represents an interesting step toward computational emotion, and may shed light to the study of emotion in virtual learning.

Summarizing the above discussion, we note that current research status shows that emotion for virtual learning is a vast area yet to be systematically explored. The following are some sample issues need to be investigated:

From a learning perspective, how many types of emotions can be distinguished?

Under which conditions certain type of emotions should be controlled and under which conditions educators can take advantage of it?

What are basic operations of emotions (such as filter out, enlargement, etc.) related to learning and how to develop computational mechanisms to support them?

Finally, just like the case of the discussion related to emergence, we may wonder whether there is a need to implement any forms of emotion at the system level in virtual learning.

\section{Learning about learners: The role of system learning}

Summarizing our discussion in the last two sections, if we agree that natural computing can make significant contributions to virtual learning, and important features such as emergence and emotions should be incorporated into the virtual learning process, then we have to provide infrastructural support in virtual learning environments. (In other words, we would like to have a positive answer for the last question appearing at the end of Section 4 and Section 5.) This can be achieved through system learning: The virtual learning environment not only provides facilities for learners, but also observes the behavior of learners and takes actions, so that its own performance can be improved (i.e., to better serve the learners). In this sense, system learning is concerned with learning about learners. (Note that the term system learning may not necessarily new to virtual learning; but it has a new meaning as it is presented here.) Among the basic functionalities of system learning are: trace activities of learners, 
apply various natural computing algorithms to support specific tasks of virtual learning (such as find a global solution or to find a solution for an individual learner), invoke appropriate operators to deal with emotions at various degrees to learners with different profiles, notify teachers to change strategies based on learners' performance, coordinate all the activities in the virtual learning environment, as well as others. Consequently, a virtual learning environment is a true human-machine symbiosis, paired by human learning and system learning.

In order to support system learning, a number of issues need to be examined. Here we list two of them. First, system learning is not just an abstract concept; it has to be implemented. It consists of selfadjustable software distributed in the entire virtual learning environment. As a second issue, we point out the important role of a system-wide database for storage and retrieval of learners' behavior, based on which various natural learning algorithms can be applied, so that new solutions can emerge. Note that the important role of database management in virtual learning environment [5] is rarely examined in literature, and this should be changed in the future.

\section{Conclusion}

In this paper we examined virtual learning in the comprehensive learning spectrum, and emphasized the importance of natural computing for virtual learning. Within this context, we also discussed important features of emergence and emotions. In addition, our introduction of natural computing into virtual learning makes the new concept of system learning necessary. This also implies the need for investigating a virtual learning architecture (or at least, a framework, in a sense similar to [11]) where human virtual learning is paired with system learning. Significant amount of work is needed for this direction of research.

\section{Acknowledgement}

This is the extended version of an earlier paper [2] appeared in International Conference of Virtual Learning (ICVL 2007). The author thanks Technical Program Committee (Chaired by Dr. Grigore Albeanu) of ICVL 2007 for recommendation of publishing this paper in IJCCC.

\section{Bibliography}

[1] A. Anohina, The terminology used in the fields of Virtual Learning, Analysis of the terminology used in the field of virtual learning, Educational Technology \& Society, Vol. 8, No. 3, pp. 91-102, 2005 (http://www.ifets.info/journals/8_3/9.pdf).

[2] Z. Chen, Learning from Nature: Natural Computing Meets Virtual Learning, Proc. 2nd Int'l Conf. Virtual Learning (ICVL 2007), pp. 117-124, 2007.

[3] D. N. Davis, Computational emergence and computational emotion, Proc. IEEE Conf. Systems, Man and Cybernetics (SMC), 1999. (http://www2.dcs.hull.ac.uk/NEAT/dnd/papers/smc99.pdf)

[4] L. N. de Castro, Fundamentals of Natural Computing: Basic Concepts, Algorithms, and Applications, Chapman \& Hall/CRC, 2006.

[5] A. Dong and H. Li, Ontology-based information integration in virtual learning environment, Proc. IEEE/WIC/ACM Int'l Conf. Web Intel. (WI'05), 2005.

[6] J. H. Holland, Emergence: From Chaos to Order, Oxford University Press, 1998. 
[7] C.-C. Huang, R. Kuo, M. Chang, J.-S. Heh, Foundation analysis of emotion model for designing learning companion agent, Proc. IEEE Int'l Conf. Advanced Learning Technologies (ICALT), pp. $326-330,2004$.

[8] S. Johnson, Emergence: The Connected Lives of Ants, Brains, Cities and Software, Penguin Books, 2002.

[9] J. LeDoux, The Emotional Brain, Simon and Schuster, 1996.

[10] M. Minsky, The Emotion Machine: Commonsense Thinking, Artificial Intelligence, and the Future of the Human Mind, Simon and Schuster, 2006.

[11] A. Nguyen, T. Nakano, T. Suda, Learning from nature: Network architecture inspired by biology, ACM Crossroads, 2004 (http://portal.acm.org/citation.cfm?id=1144389.1144391).

[12] K. O'Regan, Emotion and e-learning, Journal of Asynchronous Learning Networks, Vol. 7, No. 3, 2003.

[13] M. Poel, R. op den Akker, A. Nijholt, A. J. van Kesteren, Learning emotions in virtual environments. Proc. European Meeting on Cybernetics and Systems Research (EMCSR), 2002.

[14] P. Shutz, R. Pekrun, G. D. Phye (eds.), Emotion in Education, Academic Press, 2007.

[15] Y. Semet, E. Lutton, P. Collet, Ant colony optimisation for E-learning: observing the emergence of pedagogic suggestions, Proc. IEEE Swarm Intelligence Symposium (SIS), pp. 46 - 52, 2003.

[16] Southwest Educational Development Laboratory, How can research on the brain inform education? Classroom Campus, Vol. 3, No. 2 (http://www.sedl.org/scimath/compass/v03n02/1.html).

[17] B. van den Berg, R. van Es, R., C. Tattersall, J. Janssen, J. Manderveld, F. Brouns, H. Kurvers, R. Koper, Swarm-based Sequencing Recommendations in E-learning, Proc. 5th International Conference on Intelligent Systems Design and Applications (ISDA'05), pp. 488-493., 2005

\author{
University of Nebraska at Omaha \\ Department of Computer Science \\ Omaha, NE 68182-0500, USA \\ E-mail: zchen@mail.unomaha.edu \\ Received: November 28, 2007
}

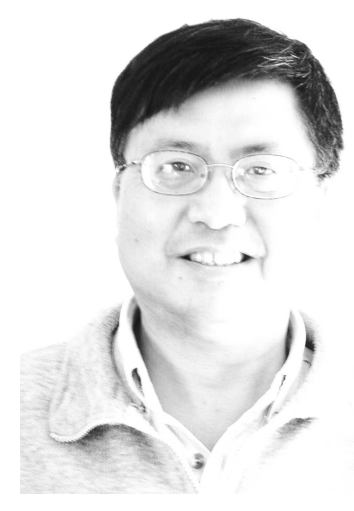

Zhengxin Chen received $\mathrm{PhD}$ degree in computer science from Louisiana State University in 1988. He is interested in a number of issues related to intelligent systems, database management systems, and data mining. Among his publications are three books: Computational Intelligence for Decision Support, Data Mining and Uncertain Reasoning: An Integrated Approach and Intelligent Data Warehousing. He also co-edited several books, includingIntelligent Agents and Their Applications in Systems Design and Data Mining and Knowledge Management. 